EPJ Web of Conferences 59, 17009 (2013)

DOI: $10.1051 /$ epjconf/20135917009

(C) Owned by the authors, published by EDP Sciences, 2013

\title{
Expansion of a plasma into vacuum with a bi-Maxwellian electron distribution function
}

\author{
A. Diaw and P. Mora \\ Centre de Physique Théorique, École Polytechnique, CNRS, 91128 Palaiseau, France
}

\begin{abstract}
A comprehensive theory is developped to describe the expansion of a plasma into a vacuum with a two-temperature electron distribution function. The characteristics of the rarefaction shock which occurs in the plasma when the hot- to the cold-electron temperature ratio is larger than 9.9 are investigated with a semi-infinite plasma. Furthermore by using a finite plasma foil, a possible heating of the cold electrons population is evidenced, for a sufficiently large hot- to the cold-electron density ratio.
\end{abstract}

\section{INTRODUCTION}

The interaction of lasers with solid targets creates plasmas which may be modeled with a two temperature electron distribution function, as has been observed by experiments [1]. The effects of these two populations of electrons on the expansion of a plasma into a vacuum and on the ion acceleration need to be clarified. In the quasi-neutral and isothermal limits, Bezzerides et al. [2] demonstrated that the self-similar model has a multivalued solution when the ratio of the temperature between the hot and the cold electrons is larger than a critical value $(\approx 9.9)$. Physically this breakdown of the self-similar model corresponds to the occurrence of a rarefaction shock wave. We first aim here to extend this work by presenting a model which gives a complete description of the rarefaction shock and its effects on the ion acceleration mechanism. On the other hand, with a kinetic model we study the time variation of the cold and hot electrons temperature in the case of a finite plasma foil.

\section{THE ELECTRON FLUID MODEL FOR PLASMA EXPANSION}

We consider at $t=0$ a 1-D plasma composed of cold ions of mass $m_{i}$ and charge $Z e$, occupying the half-space $x \leq 0$ with uniform density $n_{u} / Z$ (in the following the subscript $u$ will always correspond to the unperturbed plasma), and of electrons in equilibrium with the electric potential $\phi(x, t=0)$ which builds up due to the charge separation at the plasma edge, with density $n_{e}(\phi)$ and pressure $P(\phi)$. We assume that the potential vanishes in the unperturbed plasma $(x<0,|x| \rightarrow \infty)$, with $n_{e}(0)=n_{u}$. For $t>0$ the ions motion is described by the fluid equations, which can be written as

$$
\frac{\partial n}{\partial t}+\frac{\partial(n v)}{\partial x}=0, \quad \frac{\partial v}{\partial t}+v \frac{\partial v}{\partial x}=-\frac{e}{m} \frac{\partial \phi}{\partial x}
$$

where $n(x, t)$ is the ion density multiplied by $Z, v(x, t)$ is the ion velocity, $m=m_{i} / Z$, and where $\phi(x, t)$ is now time-dependent. Due to the smallness of their mass, the electrons are assumed to remain in equilibrium with the electric potential $\phi(x, t)$, at all positions and times. With the quasineutrality approximation, the solution corresponding to a self-similar expansion towards $x>0$ is thus given by $v=\xi+c_{s}$ where $\xi=x / t$, while the electric potential and the position $\xi$ are related by

This is an Open Access article distributed under the terms of the Creative Commons Attribution License 2.0, which permits unrestricted use, distribution, and reproduction in any medium, provided the original work is properly cited. 
the equation $[2,6]$

$$
\frac{d \xi}{d \varphi}=-\left(\frac{1}{c_{s}}+\frac{d c_{s}}{d \varphi}\right)
$$

where $c_{s}$ is the ion sound velocity. If the right-hand side of Eq. (2) is positive one obtains a multivalued solution of $\phi(\xi)$ which physically corresponds to an occurrence of a rarefaction shock, as discussed in Ref. [2]. We will construct the physical self-similar solution of the expansion of a plasma, with electrons density defined such that

$$
n_{e}(\phi)=n_{h u} \exp \left(e \phi / k_{B} T_{h}\right)+n_{c u} \exp \left(e \phi / k_{B} T_{c}\right),
$$

where $k_{B}$ is the Boltzmann constant $T_{h}$ and $T_{c}$ are respectively the hot- and the cold-electron temperatures, $n_{h u}$ and $n_{c u}$ are the corresponding densities in the undisturbed plasma.

\subsection{Rarefaction shock}

In the frame moving with the shock the equations of conservation of mass, energy and momentum flux $\operatorname{read}[2,6]$

$$
\frac{d}{d x}(n u)=0, \quad \frac{d}{d x}\left(\frac{1}{2} u^{2}+\varphi\right)=0, \quad \frac{d}{d x}\left[n u^{2}+\frac{P}{m}-\frac{\epsilon_{0} m}{2 e^{2}}\left(\frac{d \varphi}{d x}\right)^{2}\right]=0,
$$

where $u=v-\xi_{s}$ is the relative velocity and $\xi_{s}$ is the self-similar parameter corresponding to the position of the shock. The analysis of these equations allows to determine the characteristics of the rarefaction shock.

Figure 1(a) shows the profile of the electric potential corresponding to the multivalued solution of Eq. (2) for $\alpha=10^{2}$ and $y_{u}=10^{-2}$ (for convenience we have defined two dimensionless parameters $y_{u}=n_{h u} / n_{c u}$ and $\alpha=T_{h} / T_{c}$ ). Also shown in solid line is the self-similar solution obtained by using the jump conditions given by Eqs. (4) as described in Refs [2,6]. The profile can be divided as follows: the unperturbed plasma on the left of the rarefaction wave situated in $A$, a region of uniform expansion dominated by cold electrons between $A$ and $B$, the shock rarefaction joining $B$ and $E$, a plateau between $E$ and $F$, and a region of uniform expansion dominated by hot electrons on the right of $F$. The dot-dashed lines represent the results of the numerical simulations at different times $\omega_{\text {pih }} t=20$, 100 , and 500, performed with the hybrid code described in Ref. [3]. We observe an excellent agreement between the analytical model and the numerical simulations, in particular for large values of $\omega_{\text {pih }} t$. The velocity spectrum is shown in Fig. 1(b) for the same parameters as in Fig. 1(a). The spectrum is normalized to time, as the number of accelerated ions increases almost linearly with time. We recognize on the spectrum the low velocity part corresponding to the expansion dominated by the cold electrons, the dip corresponding to the shock, the peak corresponding to the plateau, and the high velocity part corresponding to the expansion dominated by hot electrons down to the velocity cut-off [5]. For ratios of densities in the unperturbed plasma $y_{u} \geq y_{B}$, where $y_{B}$ is the ratio $y_{B}=n_{h B} / n_{c B}$ calculated at point $B$ in Fig. 1(a), the rarefaction shock is directly connected to the undisturbed plasma (line A-B in Fig. 1(a) disappears). Consequently, the shock propagates inside the plasma at a supersonic velocity.

\section{THE ELECTRON KINETIC MODEL FOR PLASMA EXPANSION OF A THIN FOIL}

We also performed simulations with a non-relativistic kinetic code describing the collisionless expansion of a one-dimensional plasma slab with initial length $L$. The code is presented in detail in Ref. [4]. The electron dynamics is described by the Vlasov equation, and ions are treated as particles. The electron population is composed of a hot component and a cold component, corresponding respectively to distribution functions $f_{h}(x, v, t)$ and $f_{c}(x, v, t)$ with $f_{e}=f_{h}+f_{c}$. Moreover, the electron densities 

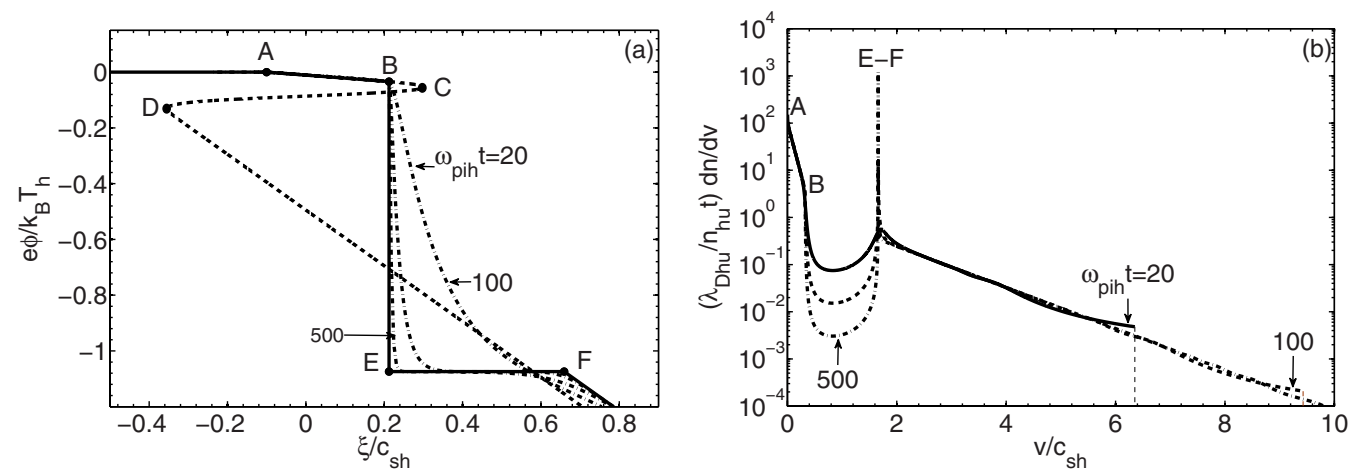

Figure 1. (a) Profile of the potential $\phi$ (dot-dashed lines), obtained with the hybrid code, as a function of $\xi$ at times $\omega_{p i h} t=20,100$ and 500, where $\omega_{p i h}=\sqrt{e^{2} n_{h u} / \epsilon_{0} m_{e}}$. The plasma parameters are $\alpha=10^{2}$ and $y_{u}=10^{-2}$. The dashed lines indicate the solution found from the numerical integration of Eq. (2). The solid line is the physical solution obtained by solving Eqs. (4) with the constraint that the flow must be sonic at point $B$. (b) normalized ion velocity spectrum as a function of time for the same parameters.

are $n_{h}(x, t)$ and $n_{c}(x, t)$, with $n_{e}=n_{c}+n_{h}$. At time $t=0$, the distribution function of each electron population is represented by a Maxwellian distribution.

In all our simulations, the ratio of the hot to the cold electron initial temperature is taken such that $\alpha \gg 1$. We define a characteristic time parameter $\tau=L / c_{s h}$ where $c_{s h}=\left(k_{B} T_{h} / m\right)^{1 / 2}$.

\subsection{Cold electrons mean temperature}

Though the distribution functions do not remain Maxwellian during the expansion, it is still possible to define a cold (and hot) electron temperature at any time and any position, with

$$
k_{B} T_{c, h}(x, t)=\frac{m_{e} \int_{+\infty}^{-\infty} v^{2} f_{c, h}(x, v, t) d v}{\int_{+\infty}^{-\infty} f_{c, h}(x, v, t) d v},
$$

where $k_{B}$ is the Boltzmann constant. A mean temperature $T_{c, h}(t)$ is defined by an integration of $T_{c, h}(x, t)$ over the simulation box.

Let us consider a plasma slab with a large value of the ratio of densities $y_{u}$, i.e., $y_{u} \gg 1$. This regime of plasma expansion is academic because it does not fit with the plasma obtained in laser plasma experiments. However, it provides interesting features of the time variation of the cold electrons energy. Figure 2(a) shows the cold electrons temperature $T_{c}(t)$ as function of time for $y_{u}=10^{4}$ and for values of $\alpha$ ranging from 10 to $10^{3}$. The expansion of the plasma slab can be separated into two phases. In the first phase $(t \lesssim 0.4 \tau)$, a rarefaction wave progresses towards the center of the foil. For time $t \gtrsim 0.4 \tau$, the plasma foil disassembles. We observe a heating of the cold electron population during the first phase. This heating mechanism increases with $\alpha$ and can be as large as a factor of $(\alpha / 3)^{2 / 3}$ for plasmas with initial width $L / 2 \lambda_{D h u} \geq 100$ [7]. This amplification results from the compression of the cold electrons by the electrostatic potential.

We develop a simple analytic model to explain these observations. One assumes that the cold electrons experience a compression inside the potential well. In this case, the time variation of the width of the cold electrons layer can be roughly estimated as $L_{c}(t) \simeq L-2 c_{s h} t$. We also consider that the cold electron density behaves as $n_{c}(t)=N_{c u} / L_{c}$, where $N_{c u}$ is the initial number of cold electrons per unit of surface. Notice that as the rarefaction wave is getting close to the target center, the density diverges. An adiabatic compression states that the relation between the pressure and electron density gives $P_{c} / n_{c}^{\gamma}=$ const, where $\gamma$ is the cold electron polytropic index. For a mono-dimensional 

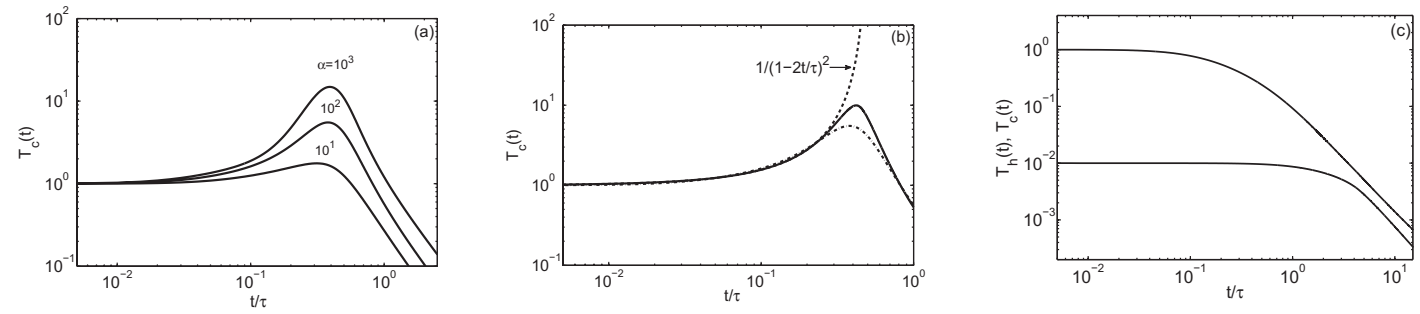

Figure 2. Cold electron mean temperature as a function of time for $y_{u}=10^{4}$. (a) $L / 2 \lambda_{D h u}=20$ and $\alpha=10-10^{3}$, (b) $\alpha=10^{2}, L / 2 \lambda_{D h u}=20$ (dashed-dot line) and $L / 2 \lambda_{D h u}=100$ (solid line). The dashed line correspond to the theoretical formula (6). $T_{c}$ is normalized with its initial value $T_{c 0}=T_{c}(0)$. (c) hot and cold electron mean temperatures normalized to hot electrons initial temperature $T_{h 0}=T_{h}(0)$ as a function of time for $\alpha=10^{2}$, $y_{u}=0.08$ and $L / 2 \lambda_{D h u}=20$.

expansion, $\gamma$ is equal to 3. By combining all these results one obtains the expression of the cold electrons temperature,

$$
T_{c}(t) \simeq \frac{T_{c 0}}{(1-2 t / \tau)^{2}},
$$

where $T_{c 0}$ is the cold electron initial temperature. The expression of the cold electron mean temperature (6) is shown in Fig. 2(b). Also shown are the results of the numerical simulations for $\alpha=10^{2}$ and $y_{u}=10^{4}$. We notice a quite good agreement in the first phase of expansion. The departure of the analytical model from the numerical results is related to the fact that when the rarefaction wave arrives at the center of the slab, the expression of the cold electron density is no longer valid.

For ratios of densities in the undisturbed plasma such that $y_{u} \lesssim 0.8 \alpha^{-1 / 2}$, the cold electrons heating vanishes as observed in Fig. 2(c). Both the cold and hot electrons cool down.

\section{CONCLUSION}

A theory of expansion of a plasma with a bi-Maxwellian electron distribution function is studied both with a fluid and a kinetic model. With an isothermal fluid model, we discussed the structure of the rarefaction shock. One distinguishes: the unperturbed plasma, a zone of rarefaction corresponding to the cold electrons expansion (this region disappears for large ratios of hot to cold electron densities), the rarefaction shock, a plateau and finally a zone of expansion dominated by the hot electrons. The expressions of the characteristics of the structure of the shock are discussed in more detail in Ref. [6].

In the second part, we studied the expansion of a plasma slab into a vacuum with initially hot and cold Maxwellian electron populations by performing simulations with a 1-D kinetic code [4], taking into account the time evolution of the electron distribution function for a finite width foil. Simulation results show that whereas hot electrons always lose energy to expanding ions, cold electrons can either gain or lose energy depending on the initial temperature ratio, density ratio, and time. When the cold electron density is not too large, they experience initially a temperature increase which may be as large as a factor of a few tens. Later on, as expected, the cold electrons eventually loose energy due to the expansion. Simple analytical calculations based on an adiabatic compression of the cold electron population by the electric field associated with the rarefaction wave confirmed the results of the numerical simulations.

\section{References}

[1] S. D. Baton et al., Plasma Phys. Control. Fusion, 47, B777 (2005)

[2] B. Bezzerides et al., Phys. Fluids 21, 2179 (1978) 
IFSA 2011

[3] P. Mora, Phys. Plasmas 12, 112102 (2005)

[4] T. Grismayer et al., Phys. Rev. E 77, 066407 (2008)

[5] P. Mora, Phys. Rev. Lett. 90, 185002 (2003)

[6] A. Diaw and P. Mora, Phys. Rev. E 84, 036402 (2011)

[7] A. Diaw and P. Mora, Phys. Rev. E 86, 026403 (2012) 